\title{
ENFERMEDAD RENAL AGUDA POR PICADURAS DE ABEJAS: REPORTE DE UN CASO
}

\section{ACUTE RENAL INJURY DUE TO BEE STINGS: A CASE REPORT.}

\section{Celso VELÁZQUEZ ${ }^{1}$, Carlos Miguel RíoS-GONZÁLEZ².}

${ }^{1}$ Hospital Regional de Ciudad del Este, Ciudad del Este, Paraguay.

${ }^{2}$ Universidad Nacional de Caaguazú, Facultad de Ciencias Médicas, Coronel Oviedo, Paraguay.

Cómo citar este artículo: Velázquez C, Ríos-González CM. Enfermedad Renal Aguda por picaduras de Abejas: Reporte de un caso. Med. clín. soc. 2019;3(3):95-99.

\section{RESUMEN}

El objetivo de la presente fue describir un caso de enfermedad renal aguda por picaduras de abejas. Se presenta un paciente masculino, de 35 años de edad, quien se encontraba consumiendo bebidas alcohólicas sin recordar cantidad, acude a extraer miel de abejas donde fue atacado por las mismas, refiere que comenzó a picarlo en la cabeza, brazos, parte anterior y posterior del tórax; Al cuarto día de su ingreso a servicio, seis días posterior del inicio del cuadro paciente se encuentra confuso, desorientado inquieto con aumento de la disnea con requerimiento de oxigeno suplementario por cánula nasal y ánurico. A pesar de la escasa publicación que se relaciona con las picaduras de abejas y el desarrollo de insuficiencia renal aguda, los hallazgos están dirigidos a la rabdomiolisis y la hemolisis como causa principal para el desarrollo de injuria renal aguda reversible. En nuestra región existe riesgo de padecer múltiples picaduras de abejas, por lo que requiere constante actualización ante un posible desenlace fatal prevenible y reversible.

Palabras clave: Venenos de Abeja; Enfermedad Crónica Renal; Abejas.

\section{ABSTRACT}

The objective of the present was to describe a case of acute renal Injuria due to bee stings. Male patient, 35 years old, who was drinking alcoholic beverages without remembering quantity, goes to a nearby lot of the house to extract honey from bees where he was attacked by them, says he began to chop it on the head, arms, front and back of the chest; On the fourth day of his admission to service, six days after the onset of the patient, he is confused, restless disoriented with increased dyspnea with a requirement for supplemental oxygen by nasal and anural cannula. Despite the limited publication related to bee stings and the development of acute renal failure, the findings are aimed at rhabdomyolysis and hemolysis as the main cause for the development of reversible acute renal injury. In our region there is a risk of multiple bee stings, so it requires constant updating to a possible preventable and reversible fatal outcome.

Keywords: Bee Venoms; Renal Insufficiency, Chronic; Bees.

Autor correspondiente: Dr. Carlos Miguel Ríos González (carlosmigue_rios@live.com). 


\section{INTRODUCCIÓN}

Las picaduras de himenópteros (abejas), con frecuencia ocurren, y por lo general no son peligrosas en personas sanas (es decir, no alérgicas), las reacciones son autolimitadas; en contraste, en personas con alergias al veneno de insectos y otros factores como el lugar y la cantidad de picaduras pueden producir reacciones severas y manifestaciones sistémicas que incluyen hasta shock anafiláctico y muerte (1). Estas reacciones producidas pueden ser inmediatas o diferidas en función del tiempo y la aparición de signos y síntomas (2).

El veneno de los himenópteros está constituido por una compleja mescla de sustancias químicas con actividades toxicas. Entre los compuestos identificados se encuentran compuestos químicos como la apamina, metilina, fosfolipasa A2 y la hialuronidasa con sus respectivas acciones tóxicas que causan reacciones adversas al picar (3).

Los signos y síntomas más frecuentes por las picaduras son varias entre los más frecuentes se mencionan oliguria, anuria, hematuria macroscópicas o microscópicas, hipotensión, enfermedad renal aguda. La recuperación de la función renal depende del manejo inicial del paciente, del inicio temprano de la terapia de sustitución renal $(4,5)$.

La causa de la insuficiencia renal aguda todo parece indicar que es la mioglobinuria debida a la rabdomiolisis por el efecto toxico de del veneno sobre los músculos, no se descarta la hemolisis como efecto que ayuda hacia el desarrollo de la falla renal, teniendo en cuenta el efecto hemolítico de algunos componentes del veneno $(6,7)$.

\section{PRESENTACIÓN DEL CASO CLÍNICO}

Paciente masculino, de 35 años de edad, tabaquista de 4 paquetes/años, bebedor ocasional, sin otros antecedentes patológicos, quien se encontraba consumiendo bebidas alcohólicas sin recordar cantidad, acude a un lugar cercano de la casa para extraer miel de abejas donde fue atacado por las mismas, refiere que comenzaron a picarlo en la cabeza, brazos, parte anterior y posterior del tórax, a su vez el paciente refiere haberse caído al suelo sin poder moverse, donde quedó en el mismo sitio por aproximadamente 3 horas, periodo durante el cual las abejas siguen atacándolo. Es trasladado por los familiares al Centro de Salud más cercano de la zona, con Presión arterial de 110/80 mmHg, Pulso: 62 latidos por minuto, temperatura de $37,4{ }^{\circ} \mathrm{C}$, frecuencia respiratoria de 20 por minuto, a su vez se encontraba severamente edematoso de cara, labios, parpados y orejas.

Se extrajeron varias abejas de la boca y de orificios nasales. A su ingreso se administraron clorfeniramina, solución fisiológica y se realizaron curaciones de sitios de picaduras. Los ojos no sufrieron ninguna alteración a pesar del severo edema de los parpados y se mantuvo bien ventilado sin oxígeno suplementario. Se mostró oligoanurico desde su ingreso con orina oscura de escasa cantidad a pesar de buena hidratación endovenosa por lo que 48 horas después fue traslado a un centro más complejos de la zona.

Exámenes de ingreso muestran: leucocitos 22880 con $90 \%$ de neutrofilos polimorfonucleares, hemoglobinas $16 \mathrm{~g} / \mathrm{dL}$, hematocrito $49 \%$ plaquetas 204000 , creatinina 4,5 mg/dL, urea $171 \mathrm{mg} / \mathrm{dL}, \mathrm{Ph} 7,35, \mathrm{PO}_{2}$ 102, $\mathrm{PCO}_{2} 24,8 \mathrm{HCO}_{3} 14$, sodio 134 , potasio 5,9 ,orina turbio, he- 
matúrico de escasa cantidad con leucocitos de 10-12 por campo, abundante cantidad de hematíes, proteínas ++, sangre ++++, TP 14,9 segundos con 76 \%, ALT 365, AST 249, BT 0,4, electrocardiograma, placa de tórax dentro de límites normales.

Cuarenta y ocho horas después paciente se encontraba decaído, áurico más edematoso, a pesar de aplicación de furosemida con leucocitos de 21 220, neutrofilos polimoefonucleares $93 \%$, hemoglobina $10,5 \mathrm{mg} / \mathrm{dL}$, hematocrito $31 \%$, plaquetas 183000 , urea $230 \mathrm{mg} / \mathrm{dL}$, creatinina $10,44 \mathrm{mg} / \mathrm{dL}, \mathrm{Ph} 7,23 \mathrm{PO}_{2} 97, \mathrm{PCO}_{2} 24,9, \mathrm{HCO}_{2}$ 10,9, sodio 137 , potasio 5,2, ecografía muestro: riñón derecho e izquierdo aumentado de tamaño con ecogenicidad aumentada, derrame pleural bilateral y presencia de ascitis.

Al cuarto día de su ingreso al servicio, seis días posterior del inicio del cuadro paciente se encuentra confuso, desorientado inquieto con aumento de la disnea con requerimiento de oxigeno suplementario por cánula nasal y ánurico. Se traslada a Unidad de Terapia Intensiva donde se inicia primera sesión de hemodiálisis y se continua cada 48 h hasta completar 8 sesiones, posteriormente espaciar cada $72 \mathrm{~h}$ y se suspende al iniciar su fase diurética. Sale del hospital a los 45 días, después en control por consultorio presenta función renal normal.

\section{DISCUSIÓN}

Los efectos de la picadura de abejas son bien conocidos, pero los mecanismos fisiopatológicos eran pocos conocidos. Desde el año 1957, a partir del cual fueron estudiados más a profundidad, cuando en un territorio de Brasil se produjo una fuga de abejas africanizadas, produciendo accidentes, algunos de ellos fatales, con desarrollo de enfermedad renal aguda (5). El veneno de las abejas es una mescla complejas de sustancias químicas con actividades toxicas. Entre los compuestos identificados se encuentran compuestos químicos como la apamina (neurotoxina, cardioestimilante, antiarritmico), metilina (hemolisis), fosfolipasa A2 (hemolisis) y la hialuronidasa (alergénico) (6).

La dosis letal media es estimada de 19 picaduras/Kg de peso. Todo niño que tenga de 10-20 picaduras y todo adulto más de 50 picaduras deben ser hospitalizados. Más de 500 picaduras se consideran potencialmente mortales. Los pacientes pueden presentar reacciones toxicas tardías o asintomáticos y sin alteraciones de los métodos auxiliares en su ingreso presentando 24 horas después signos de falla multisistemicas (7).

Las manifestaciones clínicas pueden ser: locales, que son reacciones no alérgicas donde se observa un habón eritematoso que se acompaña de dolor irritación y prurito; Sistémicas, que requiere de por lo menos de 50-100 picaduras para desarrollar cefalea, diarrea, vómitos, hipotensión, mioglobinuria, rabdomiolisis, falla renal, miocarditis, infarto de miocardio, convulsiones, coma y ocasionalmente edema cerebral, desmielinización periférica y neuropatía posterior; reacciones de hipersensibilidad, alrededor de $1 \%$ de la población es hipersensible al veneno de las abejas y para estas una sola picadura puede ser mortal; Enfermedad del suero, puede aparecer dos semanas después y se manifiesta por fiebre, artralgias, urticaria, astenia, lesiones vasculiticas, daño renal, etc (8).

Mejía-Vélez et al realizaron un estudio en 43 casos de accidentes de picaduras por abejas quienes encontraron oliguria o anuria en un $97,7 \%$, hematuria macroscópica o microscópica 
$100 \%$, hipotensión 60,9 \%. El 72,1 \% desarrollaron injuria renal aguda en las primeras 48 horas. El 86 \% requirieron diálisis para la recuperación de la funcionalidad renal que algunos llegan hasta 12 sesiones, con un promedio de 4,7. La iniciación de la diuresis comienza aproximadamente a la semana después de la picadura y la normalización de la diuresis puede prolongarse hasta las seis semanas aproximadamente, la mortalidad aproximada es de $16 \%$ $(4,9,10)$.

La mayoría de las publicaciones establece como causa de la enfermedad renal aguda la rabdomiólis (9-11), y otros postulan la hemolisis como mecanismo asociados (12,13), otros artículos proponen la toxicidad directa del veneno como mecanismo asociados a rabdomiolisis y/o a hemólisis $(14,15)$. Es importante destacar a su vez que la necrosis tubular aguda por mioglobinuria haya sido la razón de la enfermedad renal aguda.

El tratamiento actualmente es de soporte, con diálisis temprana de la enfermedad renal aguda y vigilancia hemodinámica y ventilatoria. Los pasos a seguir son: 1) la remoción temprana de los aguijones, 2) asegurar la vía aérea para la correcta ventilación y oxigenación de los pacientes, 3) tomar vía e iniciar líquidos endovenosos, 4) epinefrina por vía endovenosa o subcutánea en los pacientes que se sospechan alergia al veneno o posible reacción anafiláctica, 5) antihistamínicos y corticoesteroides endovenosos, 6) forzar diuresis con líquidos y diuréticos, 7) soporte vital en caso que lo ameriten, 8) evitar el uso de drogas neurotóxicas (16). La evolución de este paciente tuvo un comportamiento similar a una necrosis tubular aguda.

A pesar de la escasa publicación que se relaciona con las picaduras de abejas y el desarrollo de insuficiencia renal aguda, los hallazgos están dirigidos a la rabdomiólis y la hemólisis como causa principal para el desarrollo de enfermedad renal aguda reversible. En la región existe riesgo de padecer múltiples picaduras de abejas, por lo que requiere constante actualización ante un posible desenlace fatal prevenible y reversible. El traslado temprano, oportuno y el manejo multidisciplinario son fundamentales para determinar el pronóstico de estos pacientes.

\section{CONFLICTOS DE INTERÉS Y FUENTE DE FINANCIACIÓN}

Los autores declaran no poseer conflictos de interés. Fuente de financiación: ninguna.

\section{REFERENCIAS BIBLIOGRÁFICAS}

1. Matysiak J, Matysiak J, Bręborowicz A, Kokot ZJ. Diagnosis of hymenoptera venom allergy--with special emphasis on honeybee (Apis mellifera) venom allergy. Ann Agric Environ Med. 2013;20(4):875-9. URL.

2. Osuna M, Fernández-Ávila DG, Díaz MC, Gutiérrez JM. Dermatomiositis posterior a picadura de avispas. Revista Colombiana de Reumatología. 2014;21(4):232-4. https://dx.doi.org/10.1016/j.rcreu.2014.07.00

3. Marqués L. Alergia a himenópteros. Medicina respiratoria. 2010;3(2):17-26. URL. 
4. Mejía Vélez G, Mejía Vélez G. Insuficiencia renal aguda por picadura múltiple de abejas africanizadas. Comunicación de 43 casos. Nefrología. 2010;30(5):531-8. https://dx.doi.org/10.3265/Nefrologia.pre2010.May.10269

5. Schumacher MJ, Egen NB. Significance of Africanized Bees for Public Health: A Review. Arch Intern Med. 1995;155(19):2038-43. https://dx.doi.org/10.1001/archinte.1995.00430190022003

6. Hernández RV. Aspectos toxinológicos y biomédicos del veneno de las abejas Apis mellifera. latreia. 2003;16(3):217-27. URL.

7. Kolecki P. Delayed toxic reaction following massive bee envenomation. Ann Emerg Med. 1999;33(1):114-6. https://dx.doi.org/10.1016/s0196-0644(99)70428-2

8. Comité Nacional de Alergia. Guía de Práctica Clínica. Alergia a picadura de hemenópteros en pediatría. Arch Argent Pediatr. 2010;108(3):266-72. URL.

9. Humblet $Y$, Sonnet J, van Ypersele de Strihou C. Bee stings and acute tubular necrosis. Nephron. 1982;31(2):187-8. https://dx.doi.org/10.1159/000182643

10. Muñoz-Arizpe R, Valencia-Espinoza L, Velásquez-Jones L, Abarca-Franco C, GamboaMarrufo J, Valencia-Mayoral P. Africanized bee stings and pathogenesis of acute renal failure. Nephron. 1992;61(4):478. https://dx.doi.org/10.1159/000186975

11. Sert M, Tetiker T, Paydas S. Rhabdomyolysis and acute renal failure due to honeybee stings as an uncommon cause. Nephron. 1993;65(4):647. https://dx.doi.org/10.1159/000187584

12. Bresolin NL, Carvalho LC, Goes EC, Fernandes R, Barotto AM. Acute renal failure following massive attack by Africanized bee stings. Pediatr Nephrol. 2002;17(8):625-7. https://dx.doi.org/10.1007/s00467-002-0888-0

13. Gabriel DP, Rodrigues AG, Barsante RC, dos Santos Silva V, Caramori JT, Martim LC, et al. Severe acute renal failure after massive attack of Africanized bees. Nephrol Dial Transplant. 2004;19(10):2680. https://dx.doi.org/10.1093/ndt/gfh440

14. Beccari M. Dialysis or plasmapheresis for acute renal failure due to Africanized honeybee stings. Arch Intern Med. 1999;159(11):1255-6. https://dx.doi.org/10.1001/archinte.159.11.1255

15. Daher EDF, da Silva Júnior GB, Bezerra GP, Pontes LB, Martins AMC, Guimarães JA. Acute renal failure after massive honeybee stings. Rev Inst Med Trop Sao Paulo. 2003;45(1):45-50. https://dx.doi.org/10.1590/s0036-46652003000100010

16. dos Reis MA, Costa RS, Coimbra TM, Teixeira VP. Acute renal failure in experimental envenomation with Africanized bee venom. Ren Fail. 1998;20(1):39-51. https://dx.doi.org/10.3109/08860229809045088 